Article

\title{
Power Control for Device-to-Device Communication with a Hybrid Relay Mode in Unequal Transmission Slots
}

\author{
Hui Dun ${ }^{(1)}$, Fang Ye *, Shuhong Jiao and Dandan Liu \\ College of Information and Communication Engineering, Harbin Engineering University, Harbin 150001, China; \\ m18846198960@163.com (H.D.); jiaoshuhong@hrbeu.edu.cn (S.J.); liudandan@hrbeu.edu.cn (D.L.) \\ * Correspondence: yefang0923@126.com; Tel.: +86-131-5980-0815
}

Received: 19 December 2017; Accepted: 23 January 2018; Published: 31 January 2018

\begin{abstract}
Device-to-device (D2D) pairs are allowed to reuse the spectrum of cellular users who are in a good quality channel state with underlaying cellular network. However, cellular users usually suffer a poor performance in term of achievable rate when they are in a cell edge or in deep fading. To solve this problem, a hybrid relay-aided D2D communication scheme with a two-antenna infrastructure and using two unequal transmission slots is proposed in this paper. Different from the pure half-duplex and full-duplex D2D relay work, the hybrid-duplex relay mode that we propose enables the D2D relay to receive and transmit signals at the same time in the first time slot. Thus, it is similar to the full-duplex which could increase the spectrum efficiency. In addition, in the second time slot, the D2D relay will forward only the cellular user's signals, thus avoiding the transmission of mixed signals which would deteriorate the system performance, similarly to the half-duplex mode. Moreover, by bringing in a slot splitting factor, the relay node in our hybrid-duplex mode is set to guarantee the matching of the transmission rate in two hops. We formulate the problem of maximizing the D2D transmission rate while guaranteeing in priority the minimum rate for the cellular user. By using the method of rate matching and linear programming, we deduce the expression of the slot splitting factor as well as the optimal power allocation for the base station and D2D relay, while guaranteeing the minimum rate requirement for the cellular user in a close form. The simulation results show that the proposed relay-based hybrid-duplex D2D scheme outperforms the existing half-duplex and full-duplex relay-based D2D communication schemes in term of achievable rate.
\end{abstract}

Keywords: hybrid-duplex; device-to-device; relay assistant; slot splitting factor; two-antenna infrastructure

\section{Introduction}

In traditional cellular communication, cellular users transmit information through base stations to the core network. This architecture is encouraged to fulfill a ubiquitous coverage and efficient voice-oriented services [1]. However, with the explosion of local businesses, this model is awkward when it comes to communicating with users in the same cell. In addition, the increasing number of users as well as the increasing demand for high transmission rate, which result in the problem of scarce spectrum resources, are becoming a more and more prominent issue [2]. Therefore, how to use the limited spectrum and energy resources more effectively to improve the network capacity and improve users' experience has become a hot topic in recent years. In response to these challenges, many solutions have been proposed by the academia and the industry to handle this explosive growth of mobile traffic in the next-generation communication network [3]. One of them, device-to-device (D2D) communication, is considered a promising solution because it allows a user to exchange information with another user in the vicinity directly by one hop [4]. D2D communication can bring in several benefits, such as a high transmission rate, low latency, and low power consumption, which enable D2D 
communication to increase significantly the system spectral efficiency and energy efficiency. However, with the introduction of D2D users in reused mode, a further deterioration of the electromagnetic interference environment will be brought to the communication network, which will lead to the degradation of the throughput and of the quality of the service (QoS). Therefore, a study of reasonable resource allocation scheme and effective interference control mechanism has been conducted to deal with this problem, with taking into consideration of interference management, power allocation, and resource allocation [5].

The majority of the research efforts focuses on the resource allocation for cellular and D2D users in the scenario that D2D pairs reuse the spectrum of the cellular users as secondary users [6]. Various strategies have been proposed in this scenario to mitigate interference influence on cellular users as well as D2D pairs [7]. In [8], an interference-limited area control scheme has been proposed to protect D2D receivers from cellular interference, where D2D users are not allowed to share spectrum with a cellular user located in the interference-limited area where the interference-to-noise ratio for the D2D receiver is above a predetermined threshold. However, the D2D pairs reusing a cellular user's spectrum can be carried out only when the cellular users are in a good channel state quality. For the cellular users who are in the cell edge or suffer from deep fading, the communication quality will get worse because of the introduction of D2D communication. In [9], the transmit power of D2D users was restricted so that the interference inflicting cellular receivers was controlled when the D2D transmitter reused cellular resources. The sum rate of both cellular and D2D users was maximized with a minimum rate guaranteed for the cellular user in [10] for a network comprising only a single D2D pair and a single cellular user. For more practical scenarios with multiple cellular and D2D users, the spectrum and power allocation design has been considered in [11,12].

To the best of our knowledge, several works have been carried out to investigate the relay-assisted D2D communication with underlaying cellular network. By scanning existing research papers, we can classify this relay-aided D2D communication schemes into two categories in terms of relay mode, namely, half-duplex (HD) and full-duplex (FD). In fact, few works have been carried out so far to explore HD relaying-based scheme to transmit forward signals from a base station to cellular users in two transmission phases. In this scheme, respective reception and transmission at the relay are allocated to one frame which is divided into double orthogonal time slots, which are equivalent to a time-division access way for the D2D relay node [13,14]. Then, a superimposed signal, which is a linear weighted combination of the cellular user's signal and the D2D signal, is generated by the relay. The weight factor represents the power allocation for the cellular user and is a fraction of the total transmitting power of the D2D relay. Therefore, the residual power is used for broadcasting the D2D signal in the second time slot. Research efforts in [15] outline the HD mode reusing the spectrum resource allocated to the cellular user. Moreover, the D2D relay is encouraged to receive and forward signals in two equal transmission phases. Specifically, the base station broadcasts cellular signals to the cellular user and D2D transmitter which serves as a relay node in the first phase. Consequently, the achieving capacity of this direct link for the cellular user is reduced to 50 percent. To compensate for the cellular capacity loss, the D2D relay will split one fraction of the transmitting power to forward cellular signals in the second phase.

The drawback of the HD is the low efficiency in spectrum and energy. Therefore, as a replacer for the HD relay, the FD mode is more applicable in practice to transmit and receive signals simultaneously on the same frequency resource [16]. For the sake of bidirectional communication for the relay, a two-antenna infrastructure is typically deployed to facilitate spectral efficiency in practice. However, the FD relay is more susceptible to loopback interference generated from the transmitting antenna to the receiving antenna of the relay nodes [17]. Although few works have been done to eliminate the inter-relay interference, it cannot be ultimately mitigated because even the residual loopback interference is significant when compared to the white Gaussian noise [18]. Moreover, it must be made clear that because the FD mode enables the relay to forward two component signals simultaneously on the same spectral band, the cellular user's signal and D2D signal will treat each other as interference. 
As the literature [19] shows, we considered to meet the cellular users' requirements of minimum transmission rate, meanwhile maximizing the $\mathrm{D} 2 \mathrm{D}$ transmission rate. At the same time, we also took into consideration the influence of the loopback interference in the full-duplex mode. In this paper, we derive the end-to-end signal-to-noise ratio (SNR) expression of the full-duplex transmission mode and obtain the optimal value that should meet the first-order condition and second-order condition, and, finally, we get the optimal transmission power of the base station and D2D relay and the relay of the power allocation factor under a different transmission phase.

Considering the advantages and disadvantages of the above HD and FD modes, we propose a novel hybrid-duplex relay-assisted D2D communication scheme. Similar studies have been shown in [20]. In contrast to the aforementioned works, we illustrate the contributions of this paper as follows:

(1) Because of the high efficiency of the two-antenna infrastructure, we assume that the D2D relay node enables to receive and transmit signals at the same time, which represents a great improvement in spectral efficiency brought by our hybrid relay mode. However, receiving and transmitting signals synchronously generates a loopback interference from the transmitting antenna to the receiving antenna, which is inherent in the FD transmission mode. Therefore, this makes the power adaption important in terms of system performance.

(2) We transform the forwarding signals of the D2D relay from linear weighted combination signals to individual cellular signals in the second transmission phase. As we illustrate above, a mixture of cellular signals and D2D signals in the D2D relay deteriorate the respective signal-to-noise ratio (SNR) because these two kinds of signals will treat each other as interference. Consequently, a separate transmission of cellular signals and D2D signals will bring in a more efficient data delivery.

(3) Finally, we assume that the communication frame can be divided into two unequal time slots. We adopt a decode and forward (DF) mode for the D2D relay in this paper. With the use of this relay mode, the finally achievable rate for the cellular user is decided by the worse transmission hop. It should be noted that the use of two unequal time slots will lead to a more flexible rate matching among two transmission hops and yield a more efficient power allocation.

We organize this paper as follows. Section 2 presents the system model of the hybrid relay-based D2D that we proposed and the scheme of the hybrid relaying-assisted D2D communication in a cellular network. In Section 3, we formulate the objective function for of maximizing the D2D transmission rate with the constraints of the transmitting power and minimum rate requirement for cellular users. The optimal power-adaption strategy is also presented in this section. In Section 4, the evaluations for the proposed power strategy are shown versus the benchmark HD mode and FD mode proposed in [16]. Finally, some conclusions are drawn in Section 5.

\section{System Model}

The system model we consider is shown in Figure 1 and includes a macro base station, a cellular user which locates in the cell edge or suffers from deep fading, a D2D transmitter which acts as the relay, and a D2D receiver. The base station and relay node transmit the signals of the cellular user and D2D, respectively, in the first transmission phase, with a fraction of one frame simultaneously in time-domain, and reuse the cellular spectrum in frequency-domain. The residual fraction of one frame is allocated for forwarding the cellular signals by the D2D relay. It should be made clear that only one antenna is used in the second time slot rather than two antennas in our system model. We consider adopting the mode of forwarding information by using an antenna, considering that the user's power is limited and in order to avoid switching between the transmitting states and receiving states too frequently. However, this will lead to a certain waste of antenna resources. 

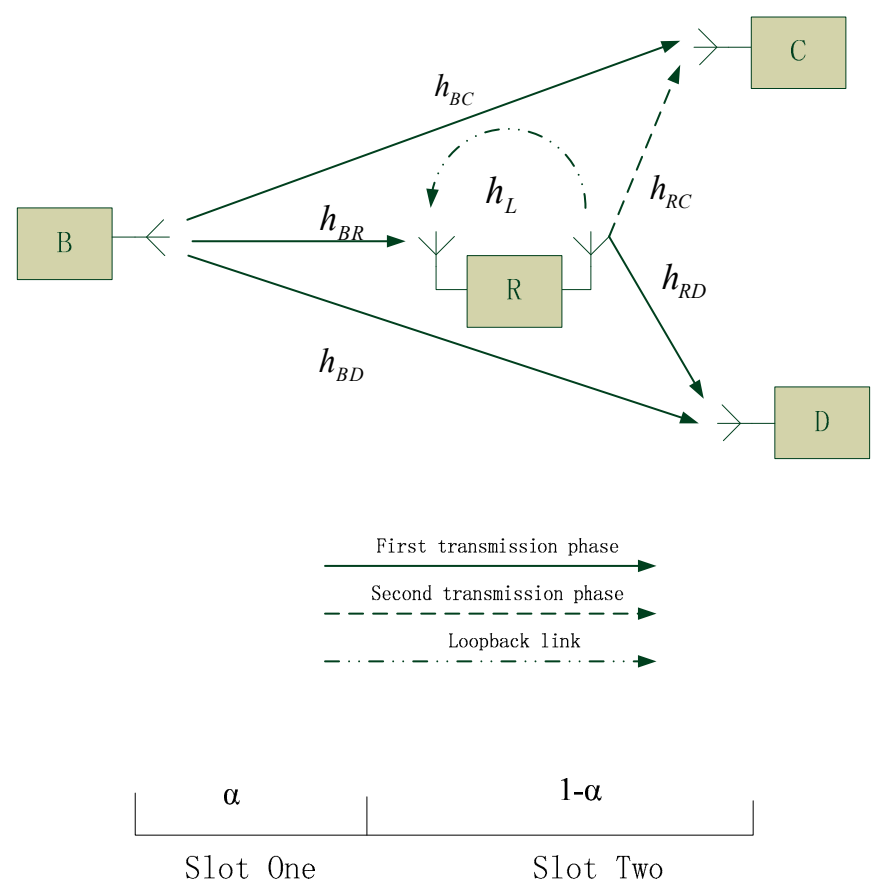

Figure 1. System model for the half-duplex (HD) relay-based device-to-device. (D2D) with slot split factor.

We assume that $\gamma_{B R}=\left|h_{B R}\right|^{2} / \sigma^{2}, \gamma_{B C}=\left|h_{B C}\right|^{2} / \sigma^{2}, \gamma_{B D}=\left|h_{B D}\right|^{2} / \sigma^{2}, \gamma_{R C}=\left|h_{R C}\right|^{2} / \sigma^{2}$, $\gamma_{R D}=\left|h_{R D}\right|^{2} / \sigma^{2}$, and $\gamma_{L}=\left|h_{L}\right|^{2} / \sigma^{2}$ denote the SNRs of the corresponding channels, where $h_{a b}$ represents the channel impulse response from node $a$ to node $b$, and $\gamma_{L}$ denotes the loopback interference of the D2D relay; $\sigma^{2}$ denotes the variance of additive white Gaussian noise (AWGN). In other words, these parameters represent the achievable SNR of corresponding channel links with usage of unit power, namely, with setting $p_{B}=1$ and $p_{R}=1$ which represent the transmit power of the base station and of the D2D relay node respectively.

In our proposed hybrid relay scheme, the D2D relay node is equipped with a two-antenna infrastructure, specifically, one receiving antenna and one transmitting antenna. Moreover, by using two unequal time slots in one frame, the D2D relay node can receive a cellular user's signals from the base station and transmit the D2D signals to the corresponding receiver synchronously in the first time slot with a portion of $\alpha$. In the second time slot, the D2D relay node uses the residual slot with a portion of $1-\alpha$ to forward the cellular user's signals solely, rather than the compounded signals, as shown in previous works.

We now describe the relaying-based D2D protocol. Let us denote $x(i)$ and $d(i)$ as the signals transmitted by the base station and the D2D relay, namely, D2D transmitter at the time instant $i$. Then we have:

$$
\begin{aligned}
& E\left\{|x(i)|^{2}\right\}=p_{B} \\
& E\left\{|d(i)|^{2}\right\}=p_{R 1}
\end{aligned}
$$

in which $E\{\cdot\}$ denotes the expectation of the variable. In addition, $p_{B}$ and $p_{R 1}$ represent the transmission power of the base station and of the D2D relay in the first phase, respectively. We consider base station and relay node use normalized transmit power. Then, Equations (1) and (2) should meet the constraints that $p_{B} \leq 1$ and $p_{R 1} \leq 1$. Furthermore, considering the loopback interference from the relay transmitting antenna to the receiving antenna, the signal $r_{R}(i)$ received at the D2D relay and the signal received at the D2D receiver $r_{D}(i)$ can be formulated as:

$$
r_{R}(i)=h_{B R} x(i)+h_{L} d(i)+n(i)
$$




$$
r_{D}(i)=h_{B D} x(i)+h_{R D} d(i)+n(i)
$$

where $n(i)$ is the AWGN with mean value $E\left\{|n(i)|^{2}\right\}=\sigma^{2}$. Thus, the signal-to-interference and noise ratios (SINR) $\gamma_{R}$ and $\gamma_{D}$ at the D2D relay and D2D receiver in the first hop can be formulated respectively as:

$$
\begin{gathered}
\gamma_{R}=\frac{p_{B}\left|h_{B R}\right|^{2}}{p_{R 1}\left|h_{L}\right|^{2}+\sigma^{2}} \\
\gamma_{D}=\frac{p_{R 1}\left|h_{R D}\right|^{2}}{p_{B}\left|h_{B D}\right|^{2}+\sigma^{2}}
\end{gathered}
$$

Next, we let the numerator and denominator be divided by $\sigma^{2}$, so the SINR can be rewritten as:

$$
\begin{gathered}
\gamma_{R}=\frac{p_{B} \gamma_{B R}}{p_{R 1} \gamma_{L}+1} \\
\gamma_{D}=\frac{p_{R 1} \gamma_{R D}}{p_{B} \gamma_{B D}+1}
\end{gathered}
$$

Then, we can calculate the achievable transmission rates for the cellular user and D2D communication, respectively, according to the Shannon's formula for the first transmission phase as:

$$
\begin{aligned}
& R_{\text {slot } 1}^{H D}=\alpha \log _{2}\left(1+\gamma_{R}\right) \\
& R_{D}^{H D}=\alpha \log _{2}\left(1+\gamma_{D}\right)
\end{aligned}
$$

As we described before, $\alpha$ represents the time duration of the first transmission slot.

In the second phase, the D2D relay decodes the cellular signal received in the first phase and forwards it to the cellular user with transmitting power $p_{R 2}$. The signal received by the cellular user's device can be expressed as:

$$
r_{C}(i)=h_{R C} \tilde{x}(i)+n(i)
$$

where $\tilde{x}(i)$ denotes the signal forwarded by the D2D relay and has the expectation value equal to $E\{\tilde{x}(i)\}=p_{R 2}$. As we have done before, the SINR of the cellular user in the second hop can be written as:

$$
\gamma_{C}=\frac{p_{R 2}\left|h_{R C}\right|^{2}}{\sigma^{2}}
$$

The expression of (12) means that the achievable rate of the second hop is determined by the transmit power and channel gain of this forwarding link. This D2D relay scheme is different from the existing related efforts which forward the combination of cellular user's and D2D signals [19]. However, the two compound signals will treat each other as interference, which leads to the deterioration of the system performance. Let the numerator and denominator be divided by $\sigma^{2}$, and (12) can be rewritten as:

$$
\gamma_{C}=p_{R 2} \gamma_{R C}
$$

It is assumed that the channel gain of all links can be estimated accurately, and we use the Shannon's formula to quantify the achievable transmission rates. Then, the achievable transmission rate for the cellular user in the second phase can be calculated as:

$$
R_{\text {slot2 }}^{H D}=(1-\alpha) \log _{2}\left(1+\gamma_{C}\right)
$$

Finally, the achievable rate for the cellular user and D2D link are given by:

$$
R_{C}^{H D}=\min \left(R_{\text {slot1 }}^{H D}, R_{\text {slot } 2}^{H D}\right)
$$




$$
R_{D}^{H D}=\alpha \log _{2}\left(1+\gamma_{D}\right)
$$

\section{Optimal Power-Adaption Strategies}

In a conventional cellular network, several cellular users may locate in the cell edge which means that they are far from the base station or are located in the shadow of huge barriers. Therefore, in this scenario, the spectrum allocated by the base station cannot deliver enough data traffic while guaranteeing the quality of service for the cellular user. This means that the achievable rate by this traditional cellular mode is lower than the minimum transmission rate required.

\subsection{Challenge of the Objective Function}

To meet the stringent requirement of a reliable communication for cellular users, a D2D pair is considered as one access point for a cellular user with reusing the spectrum resource. Therefore, in this paper, we aim to maximize the D2D transmission rate to guarantee the rate requirement of the cellular user. In this case, we define the objective function as follows:

$$
\begin{gathered}
\left(p_{B}^{*}, p_{R 1}^{*}, p_{R 2}^{*}, \alpha^{*}\right)=\underset{\left(p_{B}, p_{1}, p_{R 2, \alpha}\right)}{\arg } \alpha \log _{2}\left(1+\gamma_{D}\right) \\
\text { Subject to } R_{C}^{H D}=R_{C}^{r e} \\
p_{B} \leq 1 \\
p_{R 1} \leq 1 \\
p_{R 2} \leq 1 \\
0 \leq \alpha \leq 1
\end{gathered}
$$

where $\left(p_{B}^{*}, p_{R 1}^{*}, p_{R 2}^{*}, \alpha^{*}\right)$ represents the optimal power allocation for base station, D2D relay, and the slot splitting factor for one transmission time slot; $R_{C}^{r e}$ denotes the minimum rate requirement for the cellular user which should be strictly satisfied.

\subsection{Power Control Strategies for the System}

Next, we propose the transmission power adaptation as a technique to maximize the objective function while mitigating the effect of self-interference on the D2D relay.

Since the rate of the relayed two-hop link is limited by the worse one, the optimal transmission rate will be achieved when the SINRs of the two hops for the cellular user are equal. In other words, the transmission rate of the two hops is matching, exactly, $R_{\text {slot } 1}^{H D}=R_{\text {slot } 2}^{H D}$ which is also equivalent to $R_{C}^{r e}$. By substituting (13) to (14), we can get the optimal slot split factor and the transmit power of the relay node in the second phase by solving $R_{\text {slot } 2}^{H D}=R_{C}^{r e}$, as follows:

$$
\begin{gathered}
p_{R 2}^{*}=1 \\
\alpha^{*}=1-R_{C}^{r e} / \log _{2}\left(1+\gamma_{C}\right)
\end{gathered}
$$

Next, we will resolve the residual parameters of the system. As we can see from the objective function, the constraint of $R_{C}^{H D}=R_{C}^{r e}$ can be modified as $R_{\text {slot } 1}^{H D} \geq R_{C}^{r e}$, namely, $\alpha \log _{2}\left(1+\gamma_{R}\right) \geq R_{C}^{r e}$. It can be seen that the bottleneck of this constraint depends on the transmission in the first phase. As we described before, the relay node and base station cause mutual interference in the first phase. To get the optimal power pair $\left(p_{B}^{*}, p_{R 1}^{*}\right)$, we consider the following lemma:

Lemma 1: The function of achievable rate for hop one is monotonous increasing as the increasing of $\left(\beta p_{B}, \beta p_{R 1}\right)$, where $\beta>1$ [21]. Therefore, the optimal power allocation problem of the one-to-one pairing scheme with sharing resources will have $p_{B}^{*}=1$ or $p_{R 1}^{*}=1$. 
Proof: We will have the following deduction

$$
\begin{aligned}
\alpha \log _{2}\left(1+\frac{\beta p_{B} \gamma_{B R}}{\beta p_{R 1} \gamma_{L}+1}\right) & =\alpha \log _{2}\left(1+\frac{p_{B} \gamma_{B R}}{p_{R 1} \gamma_{L}+1 / \beta}\right) \\
& \geq \alpha \log _{2}\left(1+\frac{p_{B} \gamma_{B R}}{p_{R} \gamma_{L}+1}\right)
\end{aligned}
$$

makes sense, when we have $\beta>1$. Therefore, for the sake of maximizing the achievable rate for hop one, the optimal power pair $\left(p_{B}^{*}, p_{R 1}^{*}\right)$ will have to satisfy one of the following conditions: $p_{B}^{*}=1$ and $p_{R 1}^{*}=1$.

In the following, we can rewrite the achievable rate of hop one by substituting (7) to (9) as:

$$
R_{\text {slot } 1}^{H D}=\alpha \log _{2}\left(1+\frac{p_{B} \gamma_{B R}}{p_{R 1} \gamma_{L}+1}\right)
$$

In addition, we have $R_{\text {slot } 1}^{H D} \geq R_{C}^{r e}$ as a constraint, which means that $\alpha \log _{2}\left(1+\frac{p_{B} \gamma_{B R}}{p_{R 1} \gamma_{L}+1}\right) \geq R_{C}^{r e}$ will satisfy all pairs of $\left(p_{B}, p_{R 1}\right)$. Moreover, we change this expression in the equation as.

$$
p_{B} \geq \frac{2_{\frac{R_{C}}{\alpha}}^{R_{e}}\left(p_{R 1} \gamma_{L}+1\right)}{\gamma_{B R}}
$$

It is obvious that the power allocation for the base station and the D2D relay in the first hop forms a linear function. With the addition of the maximum power constraints, the problem of optimization for $\left(p_{B}, p_{R 1}\right)$ can be resolved with linear programming. The two kinds of feasible domains for power allocation of $\left(p_{B}^{*}, p_{R 1}^{*}\right)$ are illustrated in Figure 2. We consider the transmit power of the D2D relay $p_{R 1}$ as the vertical coordinate and the transmit power of base station as the horizontal coordinate. The feasible domain of $\left(p_{B}, p_{R 1}\right)$ is below the line $\alpha \log _{2}\left(1+\gamma_{R}\right)=R_{C}^{r e}$. The point of $p_{B}^{\min }$ denotes the minimum transmit power of the base station necessary to fulfill the rate requirement of the cellular user when the D2D communication pair cannot reuse the wireless resource. Note that we consider the constraint of rate requirement of the cellular user merely. Therefore, the optimal power will be found in the intersection of $\alpha \log _{2}\left(1+\gamma_{R}\right)=R_{C}^{r e}$ and $p_{B}=1$ or $p_{R 1}=1$, namely, point A in Figure 2. It's worth noting that because of the difference in the slope of the line, we can get two different feasible domains.

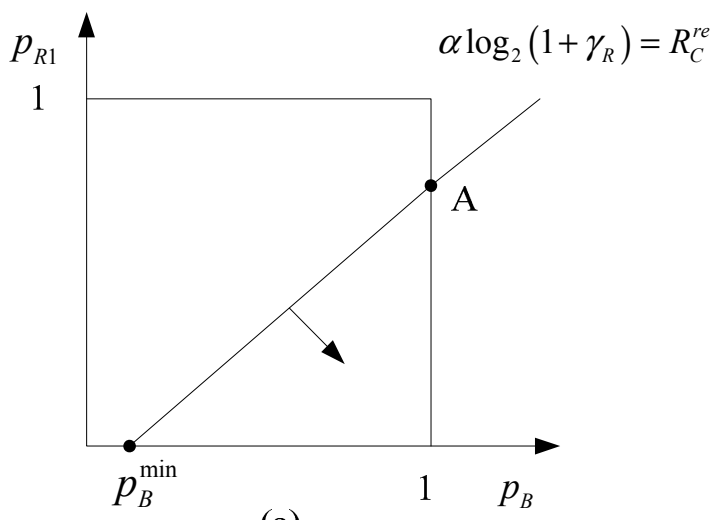

(a)

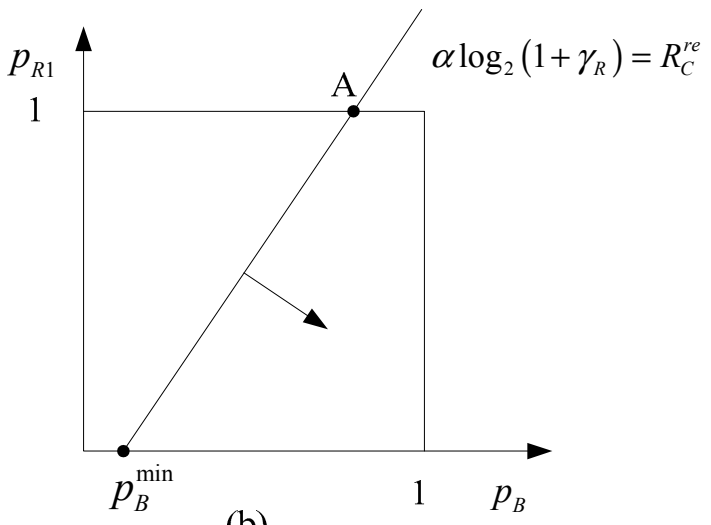

(b)

Figure 2. The feasible domain of power allocation for the base station and relay node in the first phase. 
Finally, we can get the expression of optimal power allocation of $\left(p_{B}^{*}, p_{R 1}^{*}\right)$ as:

$$
\left(p_{B}^{*}, p_{R 1}^{*}\right)=\left\{\begin{array}{c}
\left(\frac{\left(\gamma_{L}+1\right)\left(2^{R_{C}^{r e} / \alpha}+1\right)}{\gamma_{B C}}, 1\right) \\
\text { if } \alpha \log \left(1+\gamma_{B C} /\left(\gamma_{L}+1\right)\right) \geq R_{C}^{r e} \\
\left(1, \frac{\gamma_{B C}-2_{C}^{R_{C} / \alpha}+1}{\left(2^{R_{C}^{r e} / \alpha}+1\right) \gamma_{L}}\right) \\
\text { if } \alpha \log \left(1+\gamma_{B C} /\left(\gamma_{L}+1\right)\right)<R_{C}^{r e}
\end{array}\right.
$$

Until now, the proposed hybrid-duplex relay-based D2D communication system has been analyzed. In addition, the power allocation for the base station and D2D relay, and the slot split factor for different transmission phase have been obtained.

\section{Simulation Results}

In this section, we show that the simulation results demonstrate a high achievement for the transmit rate of the proposed hybrid-duplex relay-based D2D communication scheme and guarantee primarily the rate requirement of the cellular user. We choose the conventional HD relaying mode [14] and FD relaying mode proposed in [16] as the benchmark. In the simulation, we study the relaying and transmit power adaptation in the case of static channels and, thus, by exploiting instantaneous channel state information. Alternatively, the following analysis explains the performance during one instantaneous snapshot within the channel coherence time in a slow-fading environment. We assume that $\gamma_{R D}=\gamma_{R C}=10 \mathrm{~dB}$ and $\gamma_{B C}=\gamma_{B D}=-5 \mathrm{~dB}$, which correspond to the channel suffering from great path loss or deep fading. The rate requirement of the cellular user is set to $R_{C}^{r e}=0.5 \mathrm{bps} / \mathrm{Hz}$.

Figure 3 evaluates the performance of three algorithms, the novel hybrid-duplex mode we proposed, the FD mode, and the traditional HD mode, in terms of achievable transmission rate gain with different loopback interference and $\gamma_{B R}$. As we described above, the two-antenna infrastructure enables the D2D relay to receive and transmit signals simultaneously in the first phase. Moreover, the D2D relay node could forward cellular signals merely in the residual slot without mutual interference. It can be seen that the proposed hybrid-duplex mode provides a significant performance improvement when compared to the benchmark algorithms.

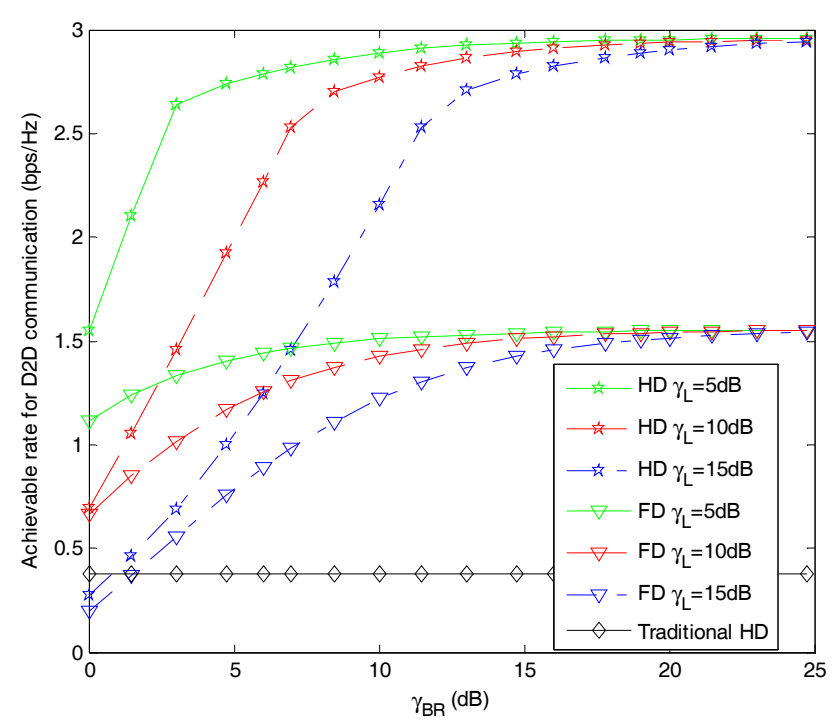

Figure 3. Rate achievement for D2D. 
In Figure 4, we evaluate the impact of the loopback interference on system performance in terms of achievable rate for D2D communication. Herein, we set the optimal value of the slot splitting factor in our proposed hybrid-duplex scheme. It can be observed that, with the increase of the loopback channel gain, the performance of both FD mode schemes and our proposed hybrid-duplex mode scheme deteriorate. This is because of the nature of the two-antenna infrastructure which enables the $\mathrm{D} 2 \mathrm{D}$ relay to receive and transmit signals at the same time. On the other hand, Figure 4 depicts that the achieved transmission rate for D2D communication with our proposed hybrid-duplex scheme is superior to the that of the conventional HD mode and FD mode.

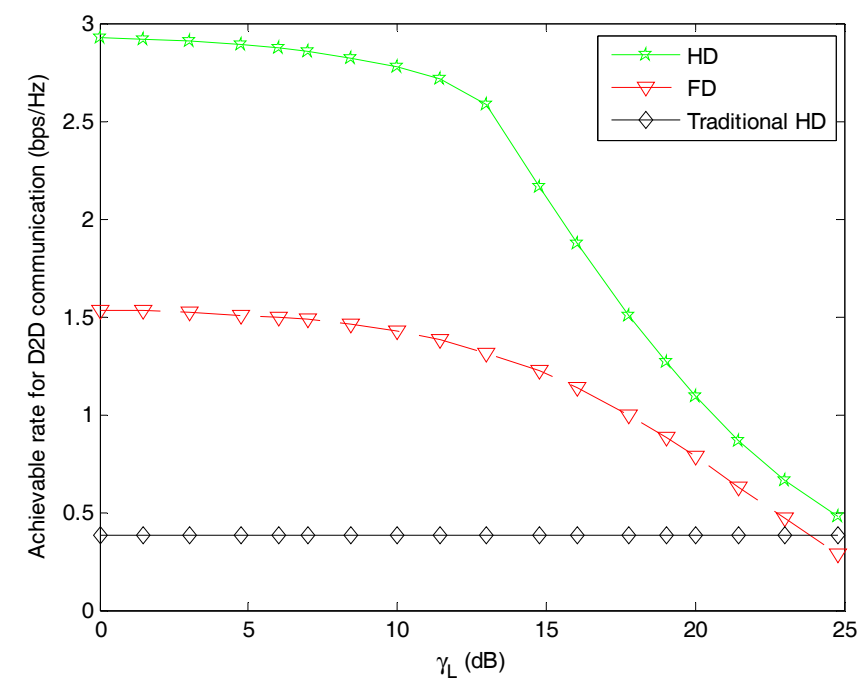

Figure 4. The performance of the D2D transmission rate with different loopback channel gains.

Figure 5 shows the performance of the relay-assisted system when the channel quality from the $\mathrm{D} 2 \mathrm{D}$ relay to the cellular user is better in the second hop. It can be observed that, with an increasing $\gamma_{R C}$, the achievable rate of D2D communication gets improved for all three kinds of relay schemes. This phenomenon can be explained as a result of the better channel state quality in the second hop, which enables the D2D relay to spare more power to forward its own signals. In addition, the proposed D2D scheme outperform the other two benchmark schemes in spite of a growth tendency of all schemes. This is because of the separate transmission of cellular signals and D2D signals in our scheme rather than a linear weighted signals with a mixture of cellular and D2D signals.

In Figure 6, we evaluate the sum transmit-power consumption of the system among the three algorithms. As we can see from Figure 6, the proposed hybrid-duplex relay mode consumes more power than the traditional HD mode and less than the FD mode. In the traditional HD mode, the D2D relay could receive and forward signals in two separate time slots. Therefore, the base station and D2D relay will use the maximum power to transmit signal. It can be seen that the sum consumed power is equal to one as the base station and the D2D relay consume the unit power at one half of frame, respectively. In addition, the FD scheme that we use as a benchmark assumes that the system will use a constant power of two. Different from these two benchmarks, the hybrid-duplex mode we propose in this paper will consume more power as $\gamma_{B R}$ increases to one particular value, and less power as $\gamma_{B R}$ increases continuously. The reason for this phenomenon is that the base station and D2D relay are forced to use more power to satisfy the transmission rate requirement and maximize the achievable rate for the D2D link. Moreover, as $\gamma_{B R}$ increases, less power is requested for the base station. It is also shown that the value of the inflexion point is greater as the channel gain of the loopback interference increases. The reason accounting for this phenomenon is that the greater the loopback channel gain, the higher the power consumption for the base station. 


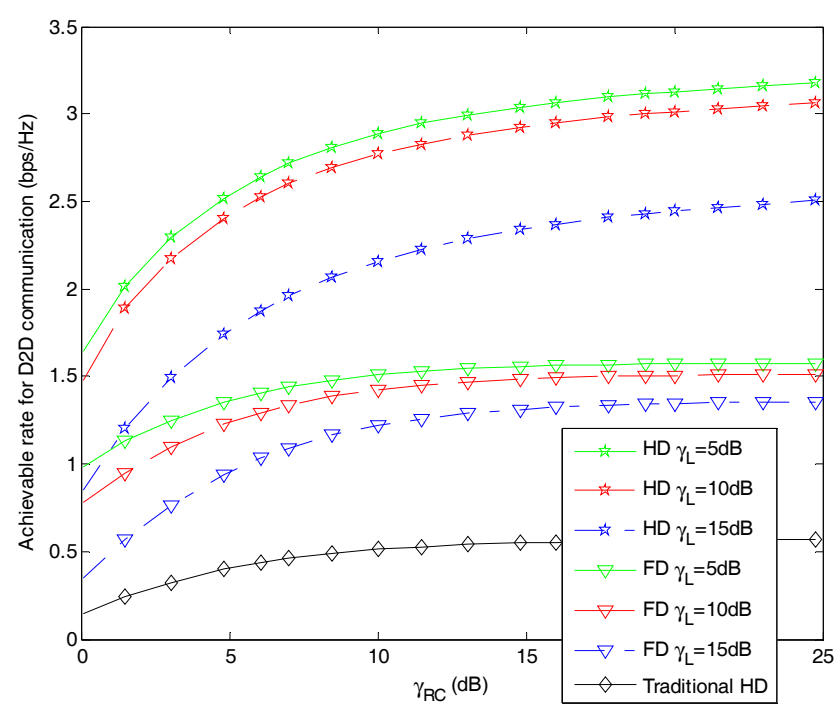

Figure 5. The effect of channel quality from the D2D relay to the cellular user.

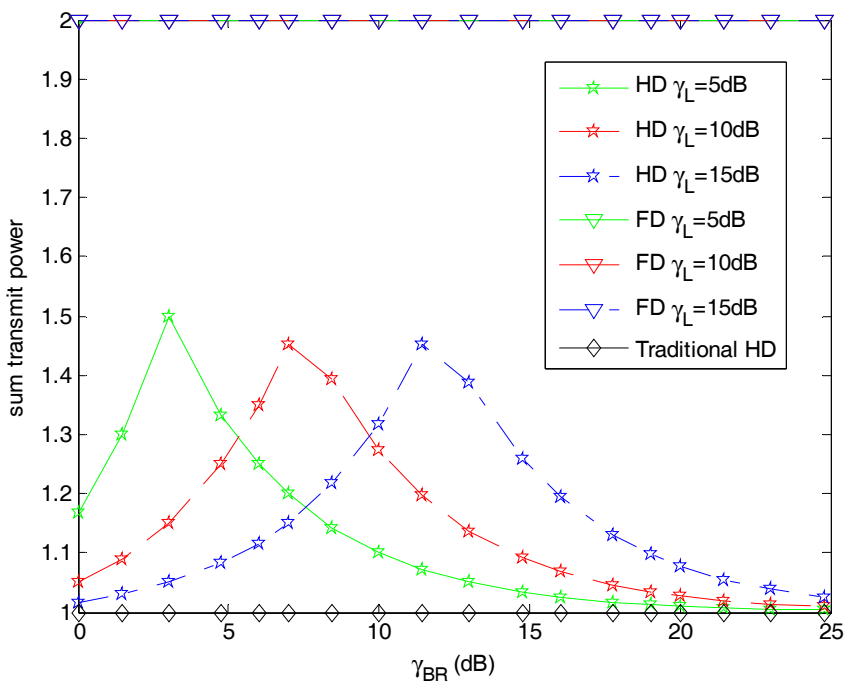

Figure 6. Sum transmit power consuming with different SINR from the base station to the D2D relay.

Furthermore, we investigate the energy efficiency of the different transmission modes in regard to $\gamma_{B R}$ in Figure 7. The simulation result reveals that the performance of the proposed relay scheme is superior to that of the traditional HD mode and FD mode. With respect to the average throughput in Figure 3 and the sum consumption of power for the system, our proposed hybrid-duplex relay mode yields the highest throughput gain when compared to the other two methods, and consumes a moderate transmit power. Consequently, the proposed hybrid-duplex relay scheme brings in a higher energy efficiency. 


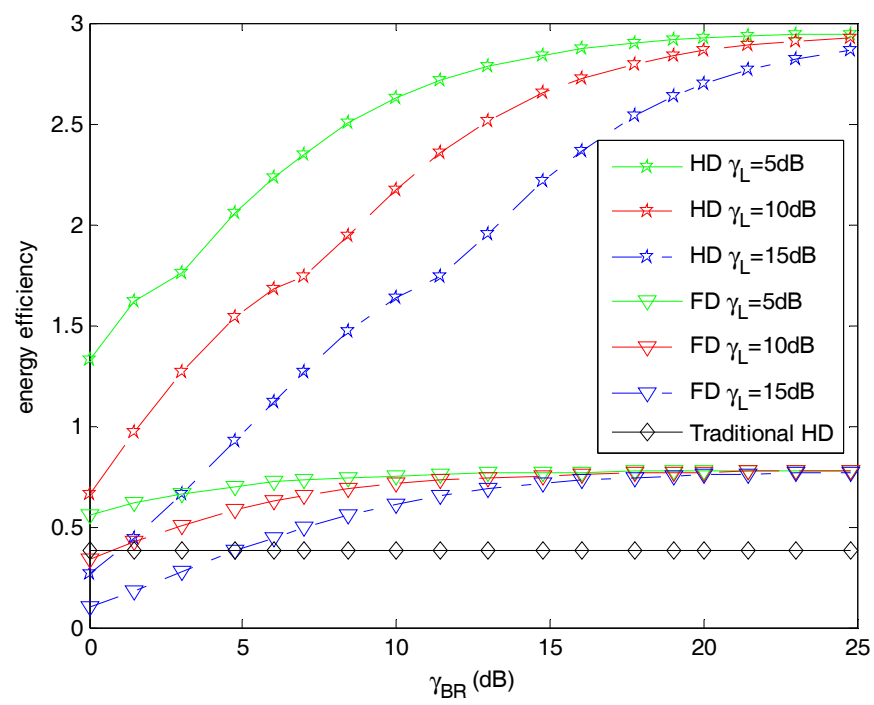

Figure 7. Spectrum efficiency of the system for different SINR from the base station to the D2D relay.

\section{Conclusions}

In this paper, we propose a novel hybrid-duplex relay-based D2D scheme with underlay downlink cellular network. Differently from the traditional HD and FD schemes, we assume the D2D relay is equipped with two antennas and transmits signals in two unequal slots. Two antennas and unequal transmission slots enable the D2D relay to receive and forward signals at the same time, which likes the FD relay mode. Moreover, in the second transmission slot, we consider adopting the mode of forwarding information by using one antenna rather than two antennas, considering that the user's power is limited and in order to avoid switching between the transmitting states and receiving states too frequently. The objective function is formulated as to maximize the transmission rate of the D2D link with a minimum SINR guaranteed for the cellular user. With the use of the matching method and linear programming, we deduce the optimal transmission power for the base station and the D2D relay while guaranteeing the minimum transmission rate for the cellular user. The simulation results show that the proposed D2D scheme can bring in more throughput gain when compared to the traditional HD and FD modes with the same system parameters. It has been proved that this relay-based D2D scheme improves the achievable rate gain for D2D users and cell-edge cellular users, with optimal slot splitting and further power allocation.

Acknowledgments: The paper was funded by the International Exchange Program of Harbin Engineering University for Innovation-oriented Talents Cultivation. This work was partially supported by the National key research and development program of China (Grant No. 2016YFF0102806), the National Natural Science Foundation of China (Grant No. 61701134), the Natural Science Foundation of Heilongjiang Province, China (Grant No. F2017004).

Author Contributions: Hui Dun conceived, designed the experiments, and performed the experiments; Fang Ye and Shuhong Jiao analyzed the data; Fang Ye contributed reagents, materials, analysis tools; Hui Dun wrote the paper. Fang Ye, Shuhong Jiao, and Dandan Liu reviewed the manuscript. All authors have read and approved the final manuscript.

Conflicts of Interest: The authors declare no conflict of interest.

\section{References}

1. Mustafa, H.; Shakir, M. Separation Framework: An enabler for cooperative and D2D communication for future 5G networks. IEEE Commun. Surv. Tutor. 2016, 18, 419-445. [CrossRef]

2. Pei, Y.; Liang, Y.C. Resource allocation for device-to-device communications overlaying two-way cellular networks. IEEE Trans. Wirel. Commun. 2013, 12, 3611-3621. [CrossRef] 
3. Niu, C.Y.; Li, Y.B.; Hu, R.Q. Fast and efficient radio resource allocation in dynamic ultra-dense heterogeneous networks. IEEE Access. 2017, 5, 1911-1924. [CrossRef]

4. Lei, L.; Zhong, Z.; Lin, C.; Shen, X. Operator controlled device-to-device communications in LTE-advanced networks. IEEE Wirel. Commun. 2012, 19, 96-104. [CrossRef]

5. Li, Y.; Song, C.; Jin, D.; Chen, S. A dynamic graph optimization framework for multihop device-to-device communication underlaying cellular networks. IEEE Wirel. Commun. 2014, 21, 52-61. [CrossRef]

6. Sun, S.H.; Gao, Q.B.; Chen, W.H.; Zhao, R.; Peng, Y. Recent progress of long-term evolution device-to-device in third-generation partnership project standardization. IET Commun. 2015, 9, 412-420. [CrossRef]

7. Wei, L.L.; Hu, R.Q.; Qian, Y.; Wu, G. Energy efficiency and spectrum efficiency of multihop device-to-device communications underlaying cellular networks. IEEE Trans. Veh. Technol. 2016, 65, 367-380. [CrossRef]

8. Min, H.; Lee, J.; Park, S.; Hong, D. Capacity enhancement using an interference limited area for device-to-device uplink underlaying cellular networks. IEEE Trans. Wirel. Commun. 2011, 10, 3995-4000. [CrossRef]

9. Yu, C.H.; Doppler, K.; Ribeiro, C.; Tirkkonen, O. Resource sharing optimization for device-to-device communication underlaying cellular networks. IEEE Trans. Wirel. Commun. 2011, 10, 2752-2763.

10. Wang, L.; Wu, H.Q. Fast pairing of device-to-device link underlay for spectrum sharing with cellular users. IEEE Commun. Lett. 2014, 18, 1803-1806. [CrossRef]

11. Doppler, K.; Rinne, M.; Wijting, C.; Ribeiro, C.; Hugl, K. Device-to device communication as an underlay to LTE-Advanced networks. IEEE Commun. Mag. 2009, 47, 42-49. [CrossRef]

12. Phunchongharn, P.; Hossain, E.; Kim, D. Resource allocation for device-to-device communications underlaying LTE-Advanced networks. IEEE Wirel. Commun. 2013, 20, 91-100. [CrossRef]

13. Zheng, D.; He, C.; Jiang, L.; Ding, J.; Zhang, Q. Power Optimization for D2D Communication Based on Rate Requirement in Relay-Assisted Networks. In Proceedings of the 2015 IEEE International Conference on Communication Workshop (ICCW), London, UK, 8-12 June 2015; pp. 686-691.

14. Han, Y.; Pandharipande, A.; Ho Ting, S. Cooperative Decode-and-Forward Relaying for Secondary Spectrum Access. IEEE Trans Wirel. Commun. 2009, 8, 4945-4950.

15. Zhou, B.; Hu, H.; Huang, S.Q.; Chen, H.H. Intracluster device-to-device relay algorithm with optimal resource utilization. IEEE Trans. Veh. Technol. 2013, 62, 2315-2326. [CrossRef]

16. Zhang, G.P.; Yang, K.; Liu, P. Power allocation for full-duplex relaying-based D2D communication underlaying cellular networks. IEEE Trans. Veh. Technol. 2015, 64, 4911-4916. [CrossRef]

17. Riihonen, T.; Werner, S.; Wichman, R. Hybrid full-duplex/half-duplex relaying with transmit power adaptation. IEEE Trans. Wirel. Commun. 2011, 10, 3074-3085. [CrossRef]

18. Kim, T.; Dong, M. An iterative Hungarian method to joint relay selection and resource allocation for D2D communications. IEEE Wirel. Commun. Lett. 2014, 3, 625-628.

19. Dun, H.; Ye, F.; Jiao, S.H. Transmission power adaption for full-duplex relay-aided device-to-device communication. Symmetry 2017, 9, 38. [CrossRef]

20. Dun, H.; Ye, F.; Jiao, S.H. Power Adaption for Half-Duplex RelayBased D2D Communication Underlaying Cellular Network. In Proceedings of the 2017 International Applied Computational Electromagnetics Society Symposium in China (ACES-China 2017), Suzhou, China, 1-4 August 2017.

21. Feng, D.Q.; Lu, L.; Yi, Y.W. QoS-aware resource allocation for device-to-device communications with channel uncertainty. IEEE Trans. Veh. Technol. 2016, 65, 6051-6062. [CrossRef]

(C) 2018 by the authors. Licensee MDPI, Basel, Switzerland. This article is an open access article distributed under the terms and conditions of the Creative Commons Attribution (CC BY) license (http://creativecommons.org/licenses/by/4.0/). 